\title{
Fragestellungen des Swiss Medical Board im Jahr 2012
}

Die Trägerschaft des Swiss Medical Board hat für das Jahr 2012 drei Analysen medizinischer Leistungen in Auftrag gegeben: die chirurgische Entfernung von Lebermetastasen bei Darmkrebs und die Anwendung von Computertomographie bei der Diagnose koronarer Herzerkrankungen. Über die Fragestellung des dritten Berichtes wird später entschieden.

Der Vorstand der Trägerschaft des Swiss Medical Board hat für das Jahr 2012 die Analyse von drei medizinischen Leistungen in Auftrag gegeben. Die Auswahl der Fragestellungen erfolgte aufgrund von Empfehlungen aus der Ärzteschaft. Zwei Analysen sollen so weit als möglich aus bereits im Ausland erstellten und veröffentlichten Berichten abgeleitet werden. Damit nimmt das Swiss Medical Board die Bestrebungen auf, Synergien zwischen den HTAAgenturen zu nutzen, wie dies auch in der internationalen Zusammenarbeit im Rahmen von SNHTA (Swiss Network for Health Technology Assessment) angestrebt wird. Ein wichtiger Bestandteil aller HTABerichte ist die medizinische Evidenz. In diesem (aufwendigen) Teil erhofft man sich die grössten Synergieeffekte. Länderspezifisch sind sicherlich die rechtlichen, ethischen und ökonomischen Erwägungen, welche das unterschiedliche Umfeld berücksichtigen und in die Empfehlungen einfliessen.

Konkret wird sich das Swiss Medical Board in diesem Jahr mit folgenden Fragestellungen befassen:

\section{Chirurgische Entfernung von Lebermetasta- sen beim kolorektalen Karzinom}

Unter dem Begriff «kolorektales Karzinom» werden die Krebserkrankungen des Dickdarms (Kolon) und des Mastdarmes (Rektum) zusammengefasst. 25 bis $35 \%$ der Patienten, bei welchen ein solches Karzinom chirurgisch entfernt wurde, erkranken im weiteren Verlauf von 5 Jahren an Metastasen in der Leber.

Im Rahmen der vorliegenden Fragestellung wird untersucht, ob eine chirurgische Entfernung solcher Metastasen im Vergleich zu anderen Therapieverfahren für die betroffenen Patienten mit einem relevanten Vorteil bezüglich Überlebensdauer und Lebensqualität verbunden ist. Hierbei werden auch die potentiell negativen Wirkungen der unterschiedlichen Therapieverfahren und das KostenWirksamkeits-Verhältnis betrachtet.

\section{Computertomographie-Scanner in der Abklärung der koronaren Herzkrankheit - eine Untersuchung auf Basis eines HTA- \\ Berichtes von NICE}

Die Koronare Herzkrankheit (KHK, Verengung der Herzkranzgefässe) ist eine häufige Erkrankung mit hoher Morbidität und Mortalität. Für die Diagnose der KHK und die Quantifizierung der Verengung stehen zwei diagnostische Möglichkeiten zur Verfügung: 1. die invasive Koronarangiographie, bei der ein Katheter über die Leistenarterie in die Herzkranzgefässe vorgeschoben wird, um die Gefässe darstellen zu können, und 2. die Computertomographie.

Bei einer Teilgruppe von Patienten mit Verdacht auf eine KHK ergibt die Computertomographie mit herkömmlichen Geräten eine zu geringe diagnostische Genauigkeit. Neue Geräte (sogenannte «NGCCT, New-generation-cardiac-CT-Geräte»), sollen diese Lücke schliessen.

Bei dieser Fragestellung wird die diagnostische Genauigkeit der neuen NGCCT-Geräte untersucht. Als Vergleich dient die Koronarangiographie. Es wird auch darauf geachtet, ob dieses Verfahren einen patientenrelevanten Vorteil bietet. Zusätzlich werden potentiell negative Wirkungen der beiden Verfahren und das Kosten-Wirksamkeits-Verhältnis betrachtet. Grundlage des Fachberichtes ist eine Evidenz-Synthese, die im August 2011 vom britischen National Institute for Health and Clinical Excellence (NICE) erstellt wurde.

Über das Thema einer dritten Fragestellung wird zu einem späteren Zeitpunkt entschieden.

Die Verabschiedung und Veröffentlichung der Berichte ist für Ende Jahr vorgesehen.

Weitere Informationen zu Fragestellungen des Swiss Medical Board: www.medical-board.ch/ index.php?id=810

\section{Swiss Medical Board}

Im Januar 2012 hat sich die Regierung des Fürstentums Liechtenstein der Trägerschaft des Swiss Medical Board angeschlossen. 